REVIEW ARTICLE

\title{
Insomnia and its management
}

\author{
M Bhatia*; M Sharma** \\ *Chairperson, Department of Sleep Medicine, ${ }^{* *}$ Clinical Psychologist,Sir Ganga Ram Hospital,New Delhi
}

Indian J Sleep Med 2009; 4.4, 125-131

\begin{abstract}
Insomnia is a perceived disturbance in the quality or quantity of sleep, which, depending on the specific condition may be associated with disturbances in objectively measured sleep. Insomnia is a common subjective complaint of inadequate sleep that affects $15 \%$ to $40 \%$ of the general population, with $10 \%$ to $15 \%$ of individuals reporting chronic insomnia of 6 month's duration.
\end{abstract}

Keyword: Insomnia

\section{Introduction}

S leep accounts for one third of human life ${ }^{1}$ and studies have suggested that poor sleep contributes to ill health. Epidemiologic studies reveal that abnormal sleep patterns predict lower life expectancy, ${ }^{2}$ and that insomnia frequently co-occurs with affective disorders, substance abuse, other physical and psychological comorbidities. ${ }^{3,4}$ Thus insomnia has become a major health-care concern over recent years because of the implications and also being a risk factors for conditions such as diabetes, hypertension and development of psychiatric disorders such as depression, anxiety and suicide. ${ }^{5,6}$ Like other common sleep disorders ${ }^{7,8,}$, insomnia with short sleep duration is also known to be associated with increased odds of diabetes ${ }^{9}$ and also increased risk of hypertension ${ }^{10}$.

There is compelling evidence that insomnia is under recognized, under diagnosed, and under treated. A longitudinal study found that practitioners in general practice settings were unaware that patients had difficulty

\section{Address for correspondence}

Dr Manvir Bhatia, MD, DM Chairperson, Department of Sleep Medicine, Senior Consultant, Department of Neurology, Sir Ganga Ram Hospital, New Delhi-60. India

Tel- $011-42251728,42251726$

Fax-011-25861002

email: manvirbhatia1@yahoo.com sleeping in more than $50 \%$ reported cases of insomnia. The inadequate identification and treatment of insomnia has significant medical and public health implications. Chronic insomnia results in impaired occupational performance and diminished quality of life, as well as higher health-care usage and costs. Employers spend more than $\$ 3,200$ more in health cost on those with insomnia than on those who sleep like a log, researchers said. (Associated Professional Sleep Societies Meeting ;June, 2007). No such data is available from our country.

As awareness of insomnia by the public continues to increase, it is important that the clinician who sees patients with sleep/wake complaints is adept in the identification, evaluation, and treatment of insomnia. ${ }^{11}$ The present article aims to provide information on the causes of insomnia, how to evaluate a patient with insomnia and the management of these patients appropriately.

\section{Definition}

Insomnia is defined as repeated difficulty with sleep initiation, duration or quality that occurs despite adequate time and opportunity for sleep with daytime complaints of sleepiness, fatigue, disturbed mood, irritability, tension headaches, gastro-intestinal (GI) complaints, worry about sleep, Generalized malaise, cognitive impairment and social/vocational dysfunction, are also common in these patients.

Indian Journal of Sleep Medicine (IJSM), Vol. 4, No. 4, 2009 


\section{Prevalence}

Insomnia is a common subjective complaint of inadequate sleep that affects $15 \%$ to $40 \%$ of the general population, with $10 \%$ to $15 \%$ of individuals reporting chronic insomnia of more than 6 month's duration. Women have 1.5 times higher risk of insomnia than men $(p<0.05)$, and the overall prevalence of insomnia increases with age. The prevalence of insomnia has been estimated at $25 \%$ in individuals aged 65 to 79 years, compared with $14 \%$ of those aged 18 to 34 years. ${ }^{12}$

\section{Classification}

A variety of approaches have been used to classify insomnia on the basis of features such as symptoms, frequency, duration, severity, and presumed etiology. ${ }^{13}$ More recent clinical definitions, such as the Research Diagnostic Criteria (RDC) for insomnia have been established by the American Academy of Sleep Medicine. The RDC is consistent with the diagnostic categories for insomnia in both the international Classification of sleep Disorders, second edition (ICSD-2) and the clinical modification of the International Classification of Diseases, 10th edition (ICD-10) (see Table 1)

Frequency, duration, and severity criteria vary considerably across different nosologies and also have limited diagnostic utility, although they may aid in identifying patients in need of treatment and/or in following up treatment response. Duration of insomnia is probably the best indicator. Insomnias are generally classified as short -term (acute or transient)- lasting days to weeks, and chronic- lasting weeks to months. Shortterm insomnia are usually due to psychological stressors (e.g., stressful life events, pain, and travel), whereas chronic insomnia is frequently associated with a variety of co morbid conditions, as well as behavioral factors that may perpetuate the insomnia. The definition of chronic ranges from duration of 2 weeks to several months, depending upon the nosology used. ${ }^{14}$

Insomnia can also be classified on the basis of etiology into primary and secondary subtypes .(See table 2) The term primary indicates that the insomnia is not caused by any known physical or mental condition, but is characterized by a consistent set of symptoms, a defined disease course, and a general responsiveness to treatment. Whereas secondary insomnia is based on the presumption that the insomnia is caused by another
Table1: Research diagnostic criteria for insomnia disorder

1. The individual reports one or more of the following sleeprelated complaints:

a. Difficulty in initiating sleep

b. Difficulty in maintaining sleep.

c. Waking up too early.

d. Sleep that is chronically non restorative or poor in quality.

2. The sleep difficulty mentioned in the preceding text occurs despite adequate opportunity and circumstances for sleep.

3.At least one of the following forms of daytime impairment related to the nighttime sleep difficulty is reported by the individual:

a. Fatigue/malaise

b. Attention, concentration, or memory impairment.

c. Social/vocational dysfunction or poor school performance.

d. Mood disturbance/irritability.

e. Daytime sleepiness.

f. Motivation/energy /initiative reduction.

g. Proneness to errors/accidents at work or while driving.

h. Tension headaches and /or GI symptoms in response to sleep loss.

i. Concerns or worries about sleep.

International Classification of Sleep Disorders, second edition (ISCD-2) and the clinical modification of the International Classification of Diseases, $10^{\text {th }}$ edition (ICD-10-CM)

Table 2: ICSD-2 Insomnia Classification

\begin{tabular}{|l|l|}
\hline 307.41 & Adjustment insomnia \\
\hline 307.42 & Psychophysiologic insomnia \\
\hline 307.42 & Paradoxic insomnia \\
\hline 307.42 & Idiopathic insomnia \\
\hline 327.02 & Insomnia caused by a mental disorder \\
\hline V69.4 & Inadequate sleep hygiene \\
\hline V69.5 & Behavioral insomnia of childhood \\
\hline 291.82 & Insomnia caused by drug or substance (alcohol) \\
\hline 292.85 & Insomnia caused by drug or substance (other) \\
\hline 327.01 & Insomnia caused by a medical condition \\
\hline 327.00 & Physiologic insomnia, unspecified \\
\hline 780.52 & $\begin{array}{l}\text { Insomnia not caused by substance or known } \\
\text { physiologic condition, unspecified. }\end{array}$ \\
\hline
\end{tabular}

International Classification of Sleep Disorders, second edition (ISCD-2) 
disorder. Many medications (see table 3), as well as a large number of psychiatric, medical, and sleep disorders, can cause or exacerbate insomnia. ${ }^{15}$

Table 3: Medications associated with insomnia

\begin{tabular}{|l|l|}
\hline Antidepressants & Decongestants \\
\hline $\begin{array}{l}\text { SSRIs (e.g., fluxetine, } \\
\text { sertraline, citalopram } \\
\text { and paroxetine) }\end{array}$ & $\begin{array}{l}\text { Phenylephrine, } \\
\text { pseudophedrine }\end{array}$ \\
\hline $\begin{array}{l}\text { SNRIs (e.g., venlafaxine } \\
\text { and duloxetine) }\end{array}$ & Hormones \\
\hline \multicolumn{1}{|c|}{ Bupropion } & Steroids, thyroid hormones \\
\hline Antihypertensives & Respiratory agents \\
\hline a- Blockers, b - Blockers & Albuterol, theophylline \\
\hline Chemotherapy & Stimulants \\
\hline Diuretics & $\begin{array}{l}\text { Amphetamone,caffeine, } \\
\text { methylphenidate, modafinil }\end{array}$ \\
\hline
\end{tabular}

SSRIs, selective serotonin reuptake inhibitors; SNRIs, serotonin nonadrenaline reuptake inbibitors

\section{How to evaluate a patient with Insomnia?}

\section{Diagnostic Evaluation (History and Examination)}

The general criteria for the diagnosis of insomnia are displayed in Table 1. It is necessary that a patient fulfill these criteria before a diagnosis of insomnia can be made.

The American Academy of Sleep Medicine recommends that all health care practitioners ask all patients about insomnia as a part of any general health screening, particularly those patients at the increased risk for sleep problems, such as women, elderly patients, and those with multiple types of psychiatric problems. A complete sleep history should be obtained, including typical routines before bedtimes, bedtime and its regularity, sleep pattern across the night, numbers and duration of awakenings, normal wake up time and its regularity, and the quality of sleep.

It is equally important to ask about daytime symptoms, including excessive daytime sleepiness and the patterns of daytime napping. Typically, individuals with insomnia may complain of daytime fatigue, but they usually don't nap excessively. Patient should also be asked about consequence of insomnia e.g., "how does your sleep problem affect your life?”

Because of higher rates of association between insomnia and medical disorders, especially psychiatric disorders, a complete medical assessment should be performed for all patients complaining of insomnia. The use of prescription and over the counter medication should be assessed because these may interfere with sleep. Many patients with insomnia tend to self medicate with alcohol, and although it can initially aid in sleep onset, alcohol tends to disrupt sleep later in the night as blood levels decline. Similarly, other substances such as caffeine, nicotine and stimulant drugs of abuse can produce insomnia. In some cases, a urine screen for toxic substances may be indicated.

Finally, patients should be asked about environmental and behavioral factors that may contribute to insomnia. Is the bed comfortable and the bedroom dark and quiet and at the appropriate temperature? Noise and heat, for example, can be highly disruptive of sleep. Behavioral factors are particularly important in chronic insomnia, because behavioral conditioning can become an important perpetuating factor in insomnia. Does the patient worry excessively about sleep and focus efforts on " trying" to sleep? Anxiety about sleep can lead to hyper arousal in the sleep setting, worsening the insomnia.

During the physical examination, the appearance of patients with insomnia may range from being hyper alert to frankly somnolent, suggesting diagnosis from primary insomnia, hyperthyroidism, abuse of stimulant drugs or anxiety disorder to sleep deprivation or sleep disorders such as sleep apnea. Hypertension, large neck circumference, enlarged tonsils, and/or narrowed oropharynx may suggest obstructive sleep apnea.(table 4)

A Sleep diary is probably the most helpful diagnostic tool in assessing insomnia, as well as following treatment response. Ideally, a patient should fill out a sleep diary for at least one week before the assessment to provide a representative picture of sleep behavior.

\section{Management}

The management of insomnia depends upon the cause and duration. In cases of secondary insomnia, those associated with SDB, RLS etc and medical illness, it is important to treat the cause and insomnia will improve consequently. However some common tools are as follows:

Indian Journal of Sleep Medicine (IJSM), Vol. 4, No. 4, 2009 
Table- 4: Diagnostic assessment of insomnia

\begin{tabular}{|l|}
\hline Assessment of sleep complaint \\
Nature of sleep problem \\
Frequency \\
Severity \\
Duration \\
Effect on daytime function \\
Predisposing factors (e.g., chronic anxiety and reactivity to stress) \\
Precipitating factors (e.g., illness and stressor) \\
Behaviors related to sleep \\
Sleep schedule, regularity. \\
Napping. \\
Maladaptive habits related to sleep \\
Exercise Patterns \\
History of prior treatment and response. \\
Medication and substance use. \\
Over the counter medications \\
Prescription medications \\
Alcohol, caffeine, tobacco \\
Recreational drugs. \\
Medical history and examinations \\
Psychiatric history \\
Screen for sleep disorders \\
Sleep apnea (e.g, presence of snoring ,obesity and excessive \\
daytime sleepiness.) \\
Restless legs/periodic limb movements (uncomfortable \\
sensations or urge to move legs when resting, Frequent kicking \\
during sleep). \\
Circadian rhythm disorder (sleep period occurring other than \\
at the desired time). \\
\end{tabular}

\section{Non Pharmacological - Psychological and Behavioral Treatments for Insomnia (see Table 5)}

There are conventional medicine, alternative medicines, psychotherapies, natural remedies, traditional medicines, as well as home remedies. The choice of the treatment method however depends on the gravity of the problem.

- Sleep Hygiene: The first step in non pharmacological treatment is the institution of proper sleep hygiene, or good sleep habits. Sleep hygiene is rarely effective when used alone, particularly in those with severe or chronic insomnia, but it makes the basis for more specific behavioral intervention. Sleep hygiene measures are intended to strengthen the physiologic
Table 5: Psychological and behavioral treatments for Insomnia

\begin{tabular}{|c|c|}
\hline Therapy & Description \\
\hline Stimulus Control therapy & $\begin{array}{l}\text { A set of instructions designed to } \\
\text { reassociate the bed/ bedroom with } \\
\text { sleep and to re-establish a } \\
\text { consistent sleep wake schedule: (1) } \\
\text { Go to bed only when sleepy; (2) } \\
\text { Get out of bed when unable to } \\
\text { sleep;(3) use the bed/ bedroom for } \\
\text { sleep only (no reading, watching } \\
\text { TV, etc), (4) arise at the same } \\
\text { time every morning;(5)no napping. }\end{array}$ \\
\hline Sleep restriction therapy & $\begin{array}{l}\text { A method designed to curtail time } \\
\text { in bed to the actual amount of } \\
\text { sleep time. For example, if a patient } \\
\text { reports sleeping an average of } 6 \\
\text { hours per night, out of } 8 \text { hour } \\
\text { spent in bed, the initial } \\
\text { recommended sleep window (from } \\
\text { lights out to final arising time) } \\
\text { would be restricted to } 6 \text { hours. } \\
\text { Periodic adjustments to this sleep } \\
\text { window are made contingent } \\
\text { upon sleep efficiency, until optimal } \\
\text { sleep duration is reached. }\end{array}$ \\
\hline Relaxation training & $\begin{array}{l}\text { Clinical procedures aimed at } \\
\text { reducing somatic tension (e.g., } \\
\text { progressive muscle relaxation, } \\
\text { autogenic training) or intrusive } \\
\text { thoughts at bedtime (e. g, } \\
\text { imaginary training meditation) } \\
\text { interfering with sleep. }\end{array}$ \\
\hline Cognitive Therapy & $\begin{array}{l}\text { Psychological methods aimed at } \\
\text { challenging and changing } \\
\text { misconceptions about sleep and } \\
\text { faulty beliefs about insomnia and } \\
\text { its perceived daytime } \\
\text { consequences. Other cognitive } \\
\text { procedures may include } \\
\text { paradoxical intention or methods } \\
\text { aimed at reducing or preventing } \\
\text { excessive monitoring of and } \\
\text { worrying about insomnia and its } \\
\text { correlates/ consequences. }\end{array}$ \\
\hline Sleep Hygiene education & $\begin{array}{l}\text { General guidelines about health } \\
\text { practices (e.g., diet, exercise, } \\
\text { substance use) and environmental } \\
\text { factors (e.g., light, noise, } \\
\text { temperature) that may promote or } \\
\text { interfere with sleep. This may also } \\
\text { includes some basic information } \\
\text { about normal Sleep and changes } \\
\text { in sleep patterns with aging. }\end{array}$ \\
\hline
\end{tabular}


processes that govern sleep. Establishing a regular bedtime and, in particular, a regular wake up time reinforces the circadian rhythm; in combination with avoiding napping, it allows for a normal build of sleep deprivation across the day to create a homeostatic need for sleep at night. Daytime light exposure and physical activity also help entrain the circadian rhythm. The bedroom environment is important in that light, noise, and heat can all disturb sleep. Sleep hygiene measures also address issues of substance use and eating behaviors may interfere with sleep. Going to bed hungry or overly full can also disturb sleep.

- Congnitive behaviour therapy (CBT) - behavioral therapy is a popular insomnia solution method. But this treatment method is exclusively for people who have sleeping problems because of some psychological disorders. But what is more important here is that this treatment method is a comprehensive one. This includes a host of individual treatments like relaxation therapy, stimulus control therapy, sleep restriction therapy as well as cognitive therapy. Behavioral therapy can be used to cure insomnia separately as well as in combination with other medications.

- Herbal remedies although not preferred by everybody are simple and effective treatments, do not have many side effects and may also result in longterm benefit. Some of the common herbal remedies used for curing insomnia are Aswagandha, Jattamamsi,Hops, Californian Poppy, Passion flower, Dog wood, and Valerian root. Another important thing about herbal treatment for insomnia is that herbs can be used in various forms like herbal tea, decoctions as well as dry powder. However, these need to be confirmed in well-controlled trials. Some reports of the beneficial effects of the herbs have been reported.

Apart from all these a serious change in lifestyle can also cure insomnia. In fact in many cases of insomnia a stressful lifestyle, which leads to depression and anxiety, is found to be the main culprit for sleeping disorders. So correcting a stressful lifestyle can also be termed as insomnia treatment. Proper eating and sleeping habits, avoiding harmful substances like drugs and alcohol are some necessary lifestyle changes required to combat the sleeping disorder.

\section{Pharmacological}

A variety of pharmacologic agents are used in the treatment of insomnia, including those tested and approved for use as hypnotics, as well as a number of prescription and over -the-counter agents that may have sedating properties but have not necessarily been approved for use as hypnotics. The ideal hypnotic should improve sleep through reducing latency to sleep onset, decreasing time awake during the sleep period, increasing total sleep without disturbing normal sleep architecture, producing improved quality of sleep and leading to better daytime functioning without hangover effects.

Studies have shown that after control for major confounders, those who reported taking sleep medication "often" or "very often: had robustly increased mortality risks: hazard ratios of 4.54 (95\% CI 2.47-8.37) in men and 2.03 (95\% CI 1.07-3.86) in women. In men, frequent hypnotic use was associated with the highest hazard for all cause mortality: larger than smoking, diabetes, heart disease, hypertension, snoring, living alone, obesity, depression, hypertension, or insomnia. Similarly, in women, regular hypnotic usage was a strong predictor for overall hazard, though not the strongest. In addition to this, controlled trials have suggested that some hypnotics can cause depression. ${ }^{17}$ Other epidemiological studies and fragmentary controlled trials have also suggested that hypnotics may cause cancer. ${ }^{18}$

All currently available drugs that have an FDA indication for the treatment of insomnia are benzodiazepine receptor agonist (BzRAs) and include both benzodiazepine and newer non-benzodiazepine drugs that not only differ in chemical structure but also bind to the benzodiazepine receptor. Other drugs that are frequently used for insomnia include antidepressants, anti psychotics, antihistamines, and anticonvulsants. From the mid 1980-90's the use of BzRAs declined by more than $50 \%$, whereas the use of sedating anti depressants increased by almost $150 \%$. By 2002, it was estimated that trazodone, a sedating antidepressant, was the most commonly prescribed drug for insomnia, and three out of the top four drugs prescribed for insomnia were anti depressants. ${ }^{19}$

\section{Benzodiazepines receptor agonist (BzRAs)}

BzRAs include the older benzodiazepines and the newer BzRAs. There are several subtypes of GABA A receptors, benzodiazepine bind to all of them with equal affinity,

Indian Journal of Sleep Medicine (IJSM), Vol. 4, No. 4, 2009 
whereas some of newer agents, including zolpidem, zaleplon and indiplon, bind preferentially to the a1 subunit of the receptor, which has been associated with hypnotic and amnestic effects but not with anxiolytic effects.

Benzodiazepines approved for the treatment of insomnia include the longer- acting agents flurazepam, temazepam, quazepam and estazolam ${ }^{20}$ and the FDA approved nonbenzodiazepine includes zolpiden, zaleplon and eszopiclone. The first two are more selective for the a1 subunit of the GABA A receptor whereas eszopiclone is less specific and also the longest half-life of these agents which is why it may be more effective for sleep maintenance; the other agents are primarily effective at sleep onset, although extended - release formulations of zolpidem and indiplon, when available may also be helpful for sleep maintenance. ${ }^{21}$

\section{Anti- depressants}

Although no anti- depressants are currently indicated for the treatment of the insomnia, in recent times they have been prescribed more frequently than BzRAs for insomnia. The most commonly used anti- depressants include trazodone, tricyclic anti- depressants (particulary amitriptyline and doxepin), and mirtazapine. When used as sleep agents, sedating anti- depressants tend to be used in doses sub therapeutic for depression. They are frequently combined with other less sedating or even activating anti- depressants.

Anti- depressants (e.g., SSRI's such fluoxetine, sertraline, paroxetne and citalopram; combined reuptake inhibitors such as venlafaxine and duloxetine; and bupropion) are commonly used to treat insomnia in patients with depression or alone in treating primary insomnia. ${ }^{22}$

\section{Antipsychotic medications}

The newer atypical antipsychotic medications particularly, quetiapine and olanzapine, are often used for their sleep -promoting effects. Their primary indication is for the treatment of schizophrenia, and they are also used as mood- stabilizing and antimaniac drugs in patients with bipolar disorders. Their primary mechanism of action is to block dopamine type 2 (D2) receptor, but sleep-inducing effect may be related to anti histamine effects and possibly to their effect on antagonism of 5- HT2C receptors. ${ }^{23}$

\section{Over-the-counter agents}

Antihistamines (usually diphenhydramine) are common active ingredients in many over -the-counter sleep aid. They act through antagonism of type 1 histamine $\left(\mathrm{H}_{1}\right)$ receptors and therefore enhance sleep by decreasing arousal due to histaminergic neuro transmission. ${ }^{24}$

Melatonin, a hormone produced by the pineal gland, is normally secreted at night. Its secretion is inhibited by light, so that it effectively indicates the length of the night across the changing seasons. Melatonin serves as a modulator of circadian rhythms and of seasonal behaviors (such as reproduction) in some animals..$^{25,26}$

Before starting any form of pharmacotherapy discuss with the patient the outcome expected, duration of use, dangers of self-medication and dose escalation and role of frequent follow-ups.

The concept of combination therapy works well in some patients particularly those with chronic insomnia due to depression. In such cases one may have to combine an anti- depressant with short acting NBzRA for initial few days, before the action of anti depressant starts.

\section{What about role of Yoga/meditation?}

They can be considered to be a part of sleep hygiene advised to all patients to ensure relaxation. These can be either practised prior to sleep (Yog Nidra) or anytime during the day.

\section{Follow up}

Patients who end up taking medications more chronically (i.e., longer than one month) should be followed up regularly to document continued efficacy of the medication, watch for dosage escalation, and monitor side effects. Setting reasonable treatment goals is important to maximize success; these should include improvement not only in various aspects of nocturnal sleep but also in daytime function. ${ }^{16}$

\section{Conclusion}

Insomnia is primarily a symptom and for appropriate and effective treatment, it is mandatory to identify the cause. There are various treatment modalities available which should be individualized for each patient. Regular follow-ups are essential to ensure compliance. Only if 
one follows the above principles can a good outcome be achieved.

\section{References}

1. Dement WC. Normal sleep, disturbed sleep, transient and persistent insomnia. Acta Psychiatr Scand Suppl. 1986; 332:41-46.

2. Kripke DF, Simnos RN, Garfinkel L, Hammond EC. Short and long sleep and sleeping pills. Is increased mortality associated? Arch General Psychiatry .1979; 36103-116.

3. Katz DA, McHorney Ca.Clinical correlates of insomnia in patients with chronic illness. Arch Intern Med.1998;158:1099-1107.

4. Taylor DJ, Licstein KL, Durrence HH. Insomnia as a health risk factor. Behav Sleep Med.2003; 1:227-247.

5. National Institutes of Health. NIH state of the science statement on manifestations and management of chronic insomnia in adults. J Clin Sleep Med 2005;1:412-21.

6. Basta M, Chrousos GP, Vela-Bueno A, Vgontzas AN. Chronic insomnia and the stress system. Sleep Med Clin 2007;2:279-91

7. Phillips B, Mannino DM. Do insomnia complaints cause hypertension or cardiovascular disease? J Clin Sleep med 2007; 3:489-94.

8. Suka M, Yoshida K, Sugimori H. Persiatent insomnia is a predictor of hypertension in Japenese male workers. J Occup helath 2003; 45:344-50.

9. Vogontzas AN, Liao D, Pejovic S, Calhoun S, Karataraki $M$, Bixler EO. Insomnia with objective short duration is associated with Type 2 diabetes: A population-based study.Diabetes Care 2009.

10. Vogontzas AN, Liao D, Bixler EO,Chrousos GP, VelaBueno A. Insomnia with objective short sleep duration is associated with a high risk for hypertension. Sleep 2009; 1;32 (8): 977-8.

11. Summers, Michael, Crisostomo, Maria I, Stepanski Edward J. Recent developments in the classification, evaluation, and treatment of insomnia .(Postgraduate Education Corner: CONTEMPORARY REVIEWS IN SLEEP MEDICINE)(Disease/Disorder overview) 1 July,2006.

12. Jadwija Najib. Eszopiclone: A Nonbenzodiazepine Sedative - Hypnotic agent for the treatment of Transient and Chronic Insomnia Clinical Therapeutics 2006;28:4.

13. Hohajen F, Kappler C, Shramn E, et. al. Sleep onset insomnia, sleep maintaining insomnia and insomnia with early morning awakening-temporal stability of subtypes in a longitudinal study on general practice attenders. Sleep $.1994 ; 17(6)$ : 551 .

14. Lichstein KL, Durrrence HH, Taylor DJ, et.al. Quantitative criteria for insomnia. Behav Res Ther.2003; 41(4): 427445.

15. Affleck G, Urrowus $\mathrm{S}$, Tennen $\mathrm{H}$, et.al. Sequential daily relations of sleep pain intensity and attention to pain among women with fibromyalgia. Pain.1996; 68(2-3);363-368.

16. Benca Ruth M. Insomnia. Avidan AY, Zee PC., eds. Handbook of Sleep Medicine, New Delhi: Wolters Kluwer Health (India);2006:36-70

17. Kripke DF. Greater incidence of depression with hypnotic use than with placebo. BMC Psychiatry 2007;7:42

18. Kripke DF. Possibility that certain hypnotics might cause cancer in skin. J Sleep Res 2008; 17:245-50.

19. Walsh JK. Drugs used to treat insomnia in 2002: Regulatory- Based rather than evidence-based medicine. Sleep .2004; 27 (8): 1441-1442.

20. Holbrook AM, Crowther R, Lotter A, et al. Meta-analysis of benzodiazepine use in the treatment of insomnia.CMAJ.2000; 162(2): 225-233.

21. Saletu -Zyhlarz G, Anderer P, Brandstatter N, et al. Placebo controlled sleep laboratory studies on the acute effects of Zolpidem on objective and subjective sleep and awakening quality in nonorganic insomnia related to neurotic and stress -related disorder. Neuropsychobiology 2000;41(3):139148.

22. Compton-McBride S, Schweitzer P, Walsh J, Most commonly used drugs to treat insomnia in 2002.Sleep.2004; 27 (suppl): A255

23. Saletu -Zyhlarz GM, Abu -Bakr MH, Anderer P, et al. Insomnia in depression :differences in objective and subjective sleep and awakening quality to normal controls and acute effects of trazodone. Prog Neuropsycopharmacol. Biol Psychiatry.2002;26 (2):249-260.

24. Sharpley AL, Vassallocm, Cowen PJ. Olanzapine increases slow - wave sleep: evidence for blockade of central 5-HT (2c) Receptors in vivo. Biol Psychiatry.2000; 47(5): 468470.

25. Witek TJ Jr, Canestrian DA, Miller RD, et al. Characterization of daytime sleepiness and psychomotor Performance following $\mathrm{H} 1$ receptor antagonist. Ann Allergy Asthma Immunol.1995; 74(5): 419-426.

26. Haimov I, Lavie $P$, Laubon $M$, et.al. Melatonin replacement therapy of elderly. Sleep. 1995; 18(7): $598-603$. 